\title{
Driver Fatigue Monitoring System Using Video Images and Steering Grip Force
}

\author{
Jun Zhao ${ }^{1,2, ~ a, ~ K u a n g r o n g ~ H a o * 1, ~ 2, ~ b ~ a n d ~ Y o n g s h e n g ~ D i n g ~}{ }^{1,2, c}$ \\ ${ }^{1}$ Engineering Research Center of Digitized Textile \& Apparel Technology, Ministry of Education, \\ Donghua University, Shanghai 201620, P. R. China \\ ${ }^{2}$ College of Information Science and Technology, Donghua University, Shanghai 201620, P. R. \\ China

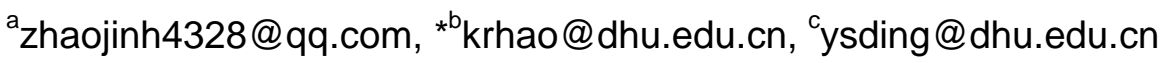

\begin{abstract}
Keywords: fatigue detection; Active Shape Models; grip force; fuzzy classifier.
Abstract. Previous research uses eye blink sensor technology to detect driver's fatigue, which has some limitations in the condition of wearing glasses and lighting changes. To overcome those problems, this paper proposes a way to detect fatigue for drivers through video images and grip forces on steering wheel. Simulated driving experiments are conducted on a platform developed by simulated driving software, during which grip forces of both hands and video images are collected. Analyzing the images from the video, we applied adaptive boosting (Adaboost) and Active Shape Models (ASM) algorithm to get the changes on the face, such as degrees of eye closure and degrees of mouth opening. These parameters including grip force that are combined using a fuzzy classifier to infer the level of inattentiveness of the driver. The results show that use of multiple visual parameters combined with steering grip force can effectively detect the driver's fatigue.
\end{abstract}

\section{Introduction}

The world vehicle population was reported to have surpassed the 1 billion-unit mark in 2010 (240 million in U.S. and 78 million in China) [1].The increasing number of car drivers accompanied with it is that more and more traffic accidents, which almost $20 \%$ of them involve driver fatigue that founded by Sleep Research Centre in UK. This exposes high life risk in accidents caused by driver's drowsiness or inattentiveness [2]. According to the U.S. National Highway Traffic Safety Administration, falling asleep while driving is responsible for at least 100000 automobile crashes annually, leading to an annual average of roughly 40000 non-fatal injuries and 1550 fatalities [3].

Driver fatigue detection techniques can be broadly classified into two categories, which include techniques that monitor the driver directly and techniques that monitor the behavior of the vehicle as a result of the driver [4].

1) Techniques that monitor the driver directly include physical and physiological data of drivers, which are used to detect their driving fatigue. These include Electroencephalography (EEG) and Electrooculography (EOG), which are the most accuracy techniques are based on physiological measures [5]. Another big part of the literature is video recognition techniques, which are using camera images have been used widely in the method because of the non-intrusive application. This approach analyses the images captured by cameras to detect physical changes in drivers, such as eyelid movement, eye gaze, yawning and head nodding [6]. Few researches on developing drowsiness detection systems based on steering grip force for drivers have been reported in the literature. Thum Chia Chieh [7], who describes an automobile driver fatigue detection method by monitoring the driver's grip force on the steering wheel, based on the variation in steering grip force due to fatigue or loosing alertness. But the model was too simple to work well. Fan Li [8], who proposes an unobtrusive way to detect driver's fatigue based on steering grip forces. The results show that variation in grip forces on steering wheel can be used to effectively detect drivers' fatigue.

2) Techniques that monitor the behavior of the vehicle consider lateral position, steering wheel movements and time-to-line crossings. These techniques are also completely non-intrusive to the 
driver, making them suitable for practical systems. However they are subject to several limitations such as vehicle type, driver experience, geometric characteristics, and condition of the road [9].

In this study, a new monitoring system has been proposed to detect subject's fatigue based on measuring eyelid movement, yawning and variation in steering grip. Advanced grip sensor technology that TekScan Grip sensor 4256E have been used here. The Grip Pressure Measurement System is a device used to measure pressure between the hand and fingers and an object. To process the images on the video, AdaBoost cascade classifier working with haar-like features being used to located the face in a frame and then we applied Active Shape Models (ASM) algorithm to get the changes on the face, such as degrees of eye closure and degrees of mouth opening. Finally, a fuzzy expert system as it makes programming easier and runs considerably faster than an equivalent sequential system and this is what we desire in our fatigue detection system. In this paper, details of technology concept and system design were described. Experiments were conducted on a platform developed by simulated driving software with different users.

Proposed System. The proposed system has two subsystems. One subsystem is for behavioral symptoms parts such as face detection, eye detection, mouth detection. And the other subsystem is for steering grip force detection part. Fig. 1 shows the proposed system whose parts will be further explained in detail.

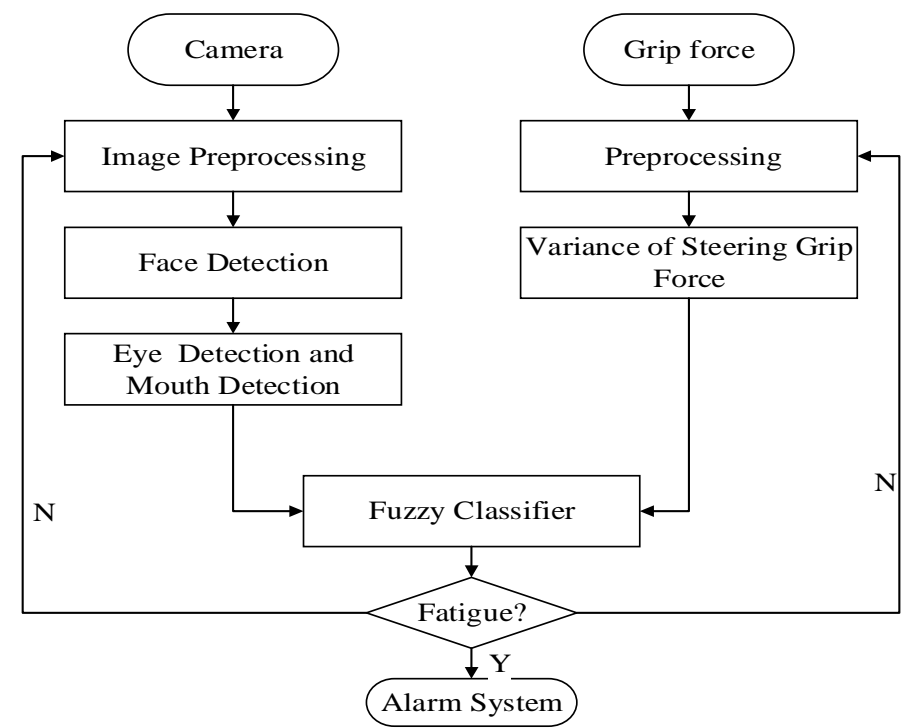

Fig. 1 Over-all system diagram

Platfrom. Platform of simulated driving system is shown in Fig. 2. On the left, a simulated steering wheel that is similar to a real one which can smoothly perform all kind of driving operation. The grip sensors are attached to the white thin cotton glove so that stability can be guaranteed. On the right, the simulated driving software can reflect the action performed by subjects. Meanwhile, video cameras are installed to record subjects' face status, which can be aided to determine whether the subjects in a drowsy state.

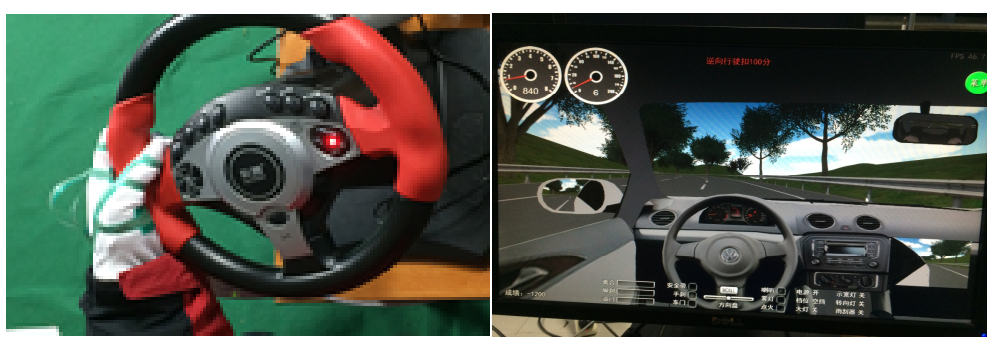

Fig. 2 Platform of simulated driving system 
Video Information. This paper mainly use Adaboost and ASM algorithm to obtain video information which includes degrees of eye closure and degrees of mouth opening. So we go a little bit deep about the two algorithms respectively in the follow.

1) adaptive boosting (Adaboost): Paul Viola and Michael[10], who firstly proposed to detect gray scale image object using haar feature which improved by Rainer Lienhart and Jochen Maydt [11], who adding tilted haar-like features into the feature set.

The face detection algorithm employs AdaBoost cascade classifier which can provide by OpenCV as haarcascade_frontalface_alt.xml file. The classifier is trained by giving positive samples that is face image and negative samples that is other image except face image before detection. The haar-like feature set which about the typical distribution of a face image is recorded in cascade classifier parameters. During detection, the captured gray scale image is split into image blocks which is decided by a scan window and then searched for faces in sequence. The distribution of each image block on the feature set is calculated and then compared with the typical distribution of face image. If most of the distribution is matched, the image block is considered to contain face image.

2) Active Shape Models (ASM): ASM who originally proposed by Cootes and Taylor [12], briefly speaking, the searching shortest Mahalanobis distance between profiles at each landmark and point distribution model (PDM). It includes the process of training and fitting. Before training, we use am_tools_win_v3 to manually mark 77 points in all images including normal and fatigue image. The 77 landmark points which introduced by Milborrow [13]. So then we can get an array of $(x, y)$ coordinates such as $\left\{\left(x_{1}, y_{1}\right),\left(x_{2}, y_{2}\right), \cdots,\left(x_{n}, y_{n}\right)\right\}$. In practice it is much more convenient to represent a shape as $2 n \times 1$ vector such as $X=\left(x_{1}, \cdots, x_{n}, y_{1}, \cdots, y_{n}\right)^{T}$ not as an array. During alignment, transforms such as scaling, rotating, and linear translation has been employed to achieve minimum distance between the shapes. The transform which translates the point $(x, y)$ by $x_{t}$ and $y_{t}$, rotates it by $\theta$, scales it by $s$ as equation (1).

$$
T_{x_{t}, y_{t}, s, \theta}\left(\begin{array}{l}
x \\
y
\end{array}\right)=\left(\begin{array}{l}
x_{t} \\
y_{t}
\end{array}\right)+\left(\begin{array}{cc}
s \cos \theta & -s \sin \theta \\
s \sin \theta & s \operatorname{con} \theta
\end{array}\right)\left(\begin{array}{l}
x \\
y
\end{array}\right)
$$

Then, Principal Component Analysis (PCA) is applied to build the model which we called point distribution model (PDM). The shape model includes an average face and allowed distortions of the average face, which can be described in equation (2).

$$
X=\bar{X}+P b
$$

$X$ is the generated shape vector, $\bar{X}$ is the mean shape, $P$ is the matrix of limited number eigenvectors corresponding to the largest eigenvalues of the covariance matrix of the training shape points, $b$ is a vector of weights. During training, a model for each landmark is built by creating a mean profile $\bar{g}$ and a covariance matrix $S_{g}$ of all training profiles (one from each image) at that landmark. During fitting, at each landmark we form several search profile by sampling the image in the neighborhood of the landmark. The distance between a search profile and the model mean profile is calculated using the Mahalanobis distance $d$ in equation (3). The process is repeated for each landmark.

$$
d=(g-\bar{g})^{T} S_{g}^{-1}(g-\bar{g})
$$

Finally, ASM is used to obtain the 77 landmarks in Fig. 3. 


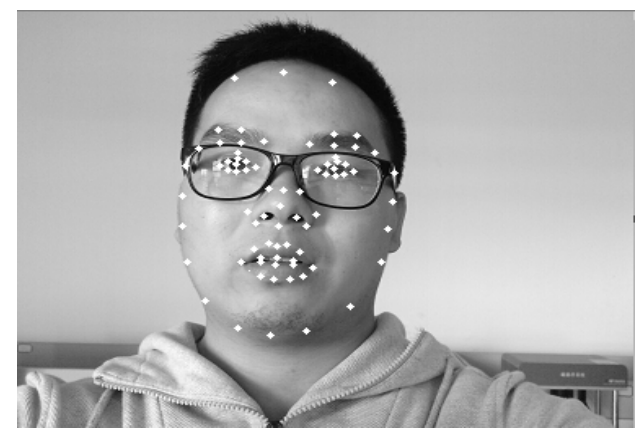

Fig. 3 The 77 landmarks

3) Openness definition: To overcome scale variability, we use the ratio of height and width to define openness in Fig. 4, which is one frame we recorded in a video. For eyes, the height is the distance between the upper-eyelid point and bottom-eyelid point and the width is the distance between the left corner point and the right corner point of the eye. For mouth, the height is the distance between the point of top mouth lips and the point of bottom mouth lips and the width is the distance between the left corner and the right corner point of the mouth.

Grip Force Information. Previous study shows that the characteristic of variation in steering grip force is due to fatigue or loosing alertness [7-8, 14]. Experiments with grip sensors (TekScan Grip sensor 4256E) attached to the hands (shown in Fig. 5) Will be carried out to evaluate correlation between grip strength and driving fatigue in a quantitative manner. On the right, the results presented in a $2 \mathrm{D}$ image way. System configuration for the sample frequency needs to set $20 \mathrm{~Hz}$ so we can collect drivers' grip force response to a video signal identically.

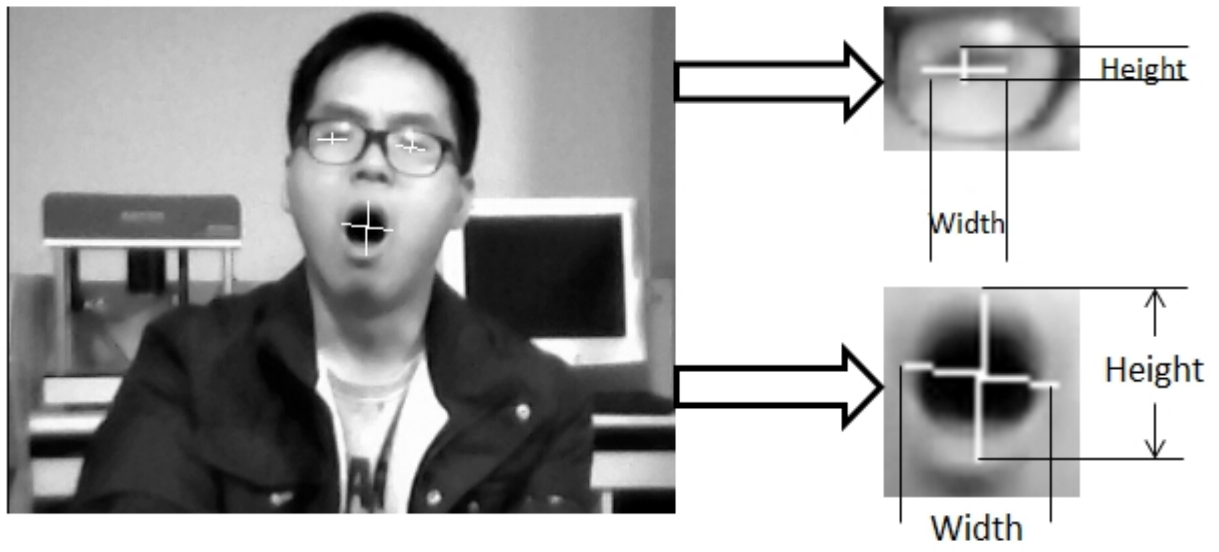

Fig. 4 Definition of openness using ratio of height and width

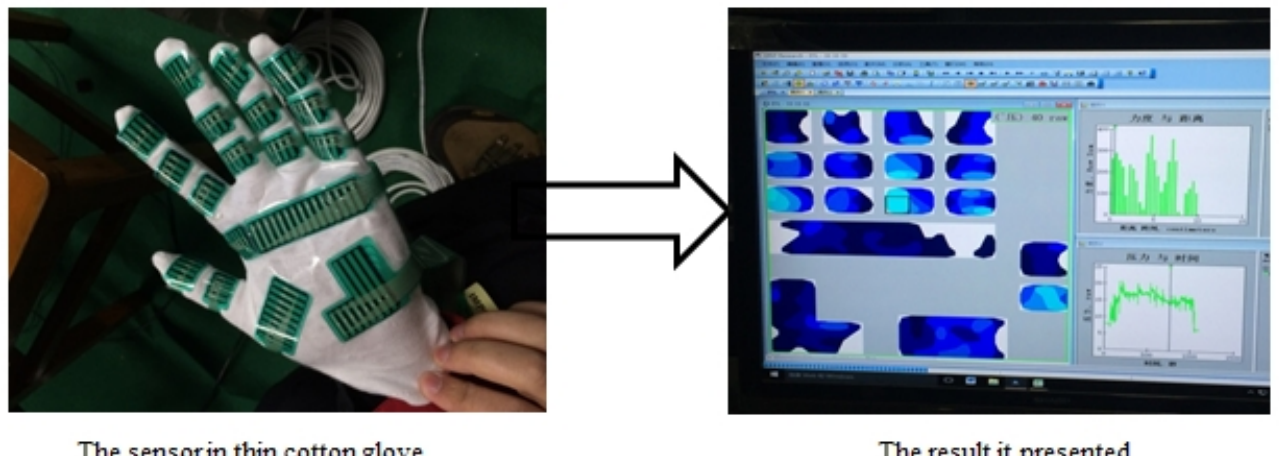

Fig. 5 TekScan grip sensor 4256E attached to the hand and result presented on the right

Driver fatigue Computation. Because human fatigue is a very complicated process, which is not observable and can only be inferred by available information. It is not easy to represent it as quantitative value from multiple sources into one representative format. So we used the fuzzy set to 
estimate fatigue with various parameters obtained in the precious section. Fuzzy inference system has a reputation for its well-known linguistic concept modeling ability and can be used for knowledge induction processes [15].

Frist of all, we need to define number and nature of the variables involved in the diagnosis process according to the domain expert experience. This paper proposes several variables, which include degrees of eye closure, degrees of mouth opening, and steering grip force. The fuzzy inputs and output are normalized and their corresponding fuzzy sets and linguistic terms are shown in Table 1.

We can get fuzzy rules based on the previous defined variables. These rules are of the form "If condition, Then conclusion," where both the premise and conclusion use the linguistic terms previously defined, as in the following example:

1) If Degrees of eye closure is small and Degrees of mouth opening is small and Variance of steering grip force is small, then "Driver fatigue level" is small.

2) If Degrees of eye closure is large and Degrees of mouth opening is large and Variance of steering grip force is large, then "Driver fatigue level" is large.

\begin{tabular}{|l|l|l|l|c|}
\multicolumn{4}{c}{ Table1 } & FUZZY VARIABLES \\
\hline \multicolumn{1}{|c|}{ Variable } & Type & Range & Labels & Linguistic terms \\
\hline $\begin{array}{l}\text { Degrees of eye } \\
\text { closure }\end{array}$ & In & {$[0.0,1.0]$} & 5 & small, medium small, medium, medium large, large \\
\hline $\begin{array}{l}\text { Degrees of mouth } \\
\text { opening }\end{array}$ & In & {$[0.0,1.0]$} & 5 & small, medium small, medium, medium large, large \\
\hline $\begin{array}{l}\text { Variance of steering } \\
\text { grip force }\end{array}$ & In & {$[0.0,1.0]$} & 3 & small, medium, large \\
\hline Driver fatigue level & Out & {$[0.0,1.0]$} & 5 & small, medium small, medium, medium large, large \\
\hline
\end{tabular}

\section{Experiment Results.}

Test Squence and Recorded steering grip force. The system has been tested in the laboratory with video and steering grip force recordings. The video sequences were recorded with a resolution of $640 \times 480$ pixels at 20 frames per second. The camera mainly focuses on the frontal face. Four experimental subjects have been tested in the simulated driving environment, which two of them wearing glasses named User1 and User3. A thorough frame by frame investigation has been conducted to verify the accuracy, which is stated in Table 2 . The average accuracy is $99.42 \%$.We choose a representation 795 frames fatigue clip which the PERCLOS is above 0.15 within the longest closure time duration [15] to demonstrate the transition from normal to fatigue. Figs 6 and 7 are off-line processed results for the percentage of openness of the eyes and mouth. For steering grip force, we obtained it from the TekScan Grip pressure measurement system, and then calculated the variance every 0.1 second until the end of the data as shown in Fig. 8.

Parameter Computation. Because the stipulation of human fatigue is a very complicated process, we used the fuzzy set to estimate fatigue with various parameters. This system can compute several parameters. They include degrees of eye closure, degrees of mouth opening and variance of steering grip force. Fig. 9depicts the parameters measured and shows the driver fatigue level. Compare with the combination of eye closure and mouth opening, the three parameters that is eye closure mouth opening and variance of steering grip force show much more robust performance. According to the results, we can divide it into three states such as $0.1-0.3$ is normal driving state, $0.3-0.5$ represents minor fatigue and anything above 0.5 is fatigue and send the alarm.

Table2 SIMULATED DROWSINESS FRAMES

\begin{tabular}{ccccc}
\hline Experimental Subjects & Tested Frames & Correct Frames & Failure Frames & Accuracy \\
\hline User1(wearing glasses) & 795 & 789 & 6 & $99.24 \%$ \\
User2 & 2000 & 1989 & 11 & $99.45 \%$ \\
User3(wearing glasses) & 900 & 895 & 6 & $99.44 \%$ \\
User4 & 650 & 647 & 3 & $99.53 \%$ \\
\hline
\end{tabular}




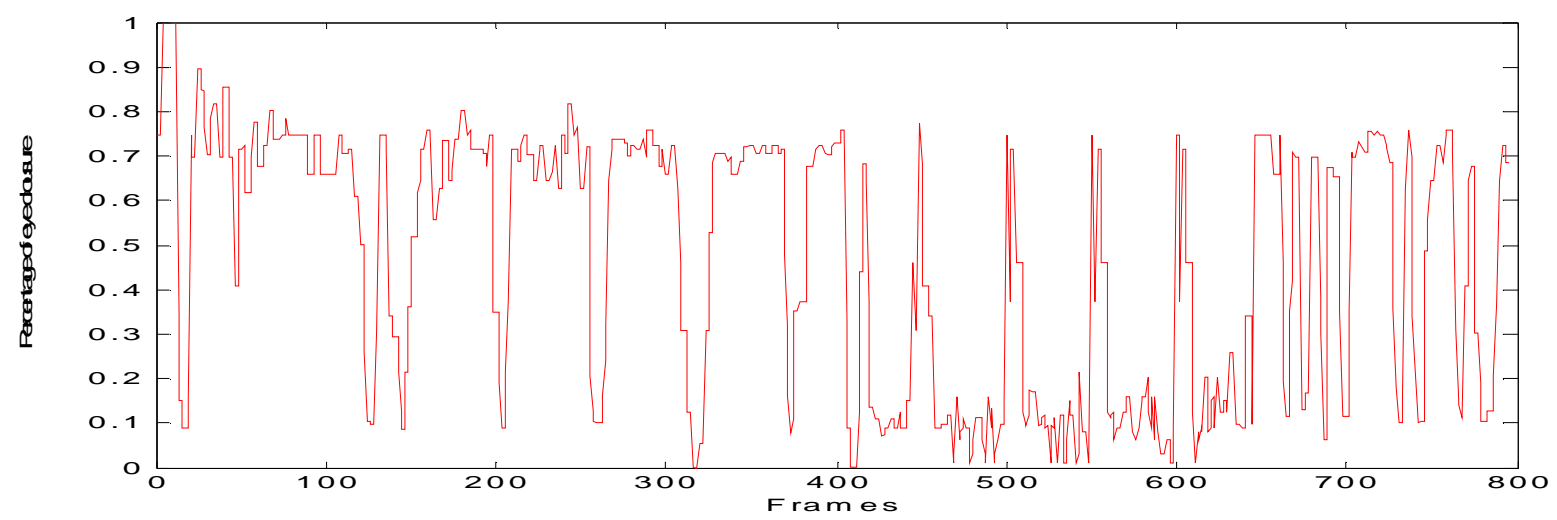

Fig. 6 Percentage of eye closure

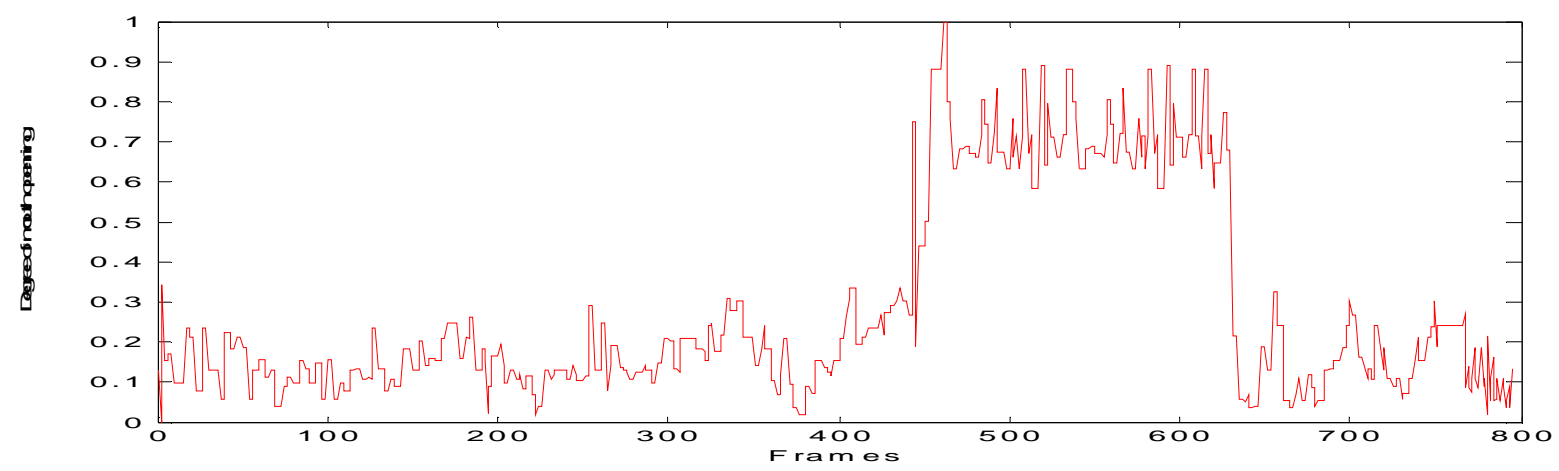

Fig. 7 Percentage of mouth opening

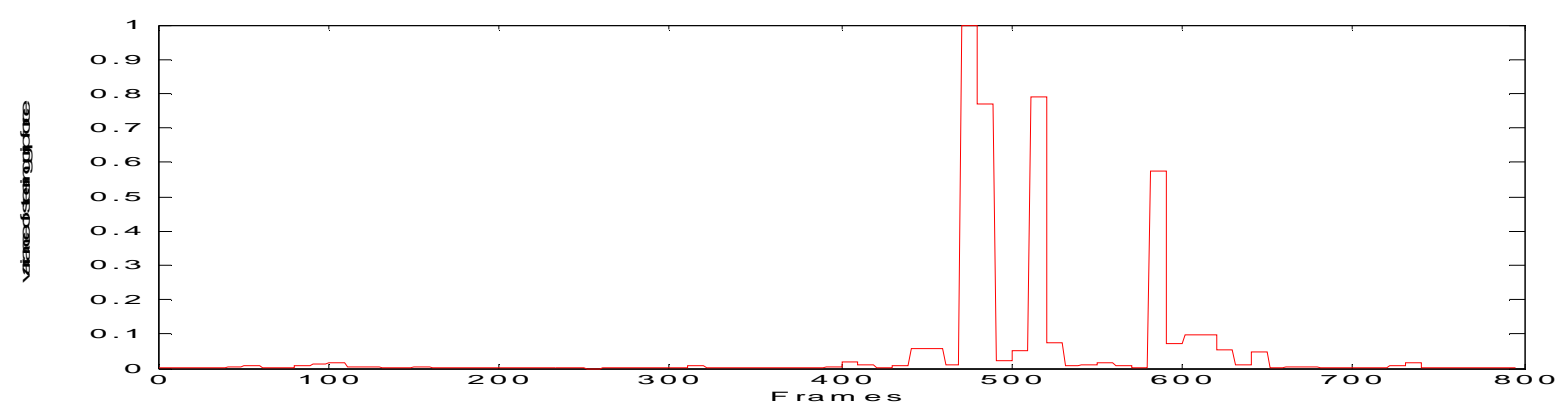

Fig. 8 Variance of the steering grip force

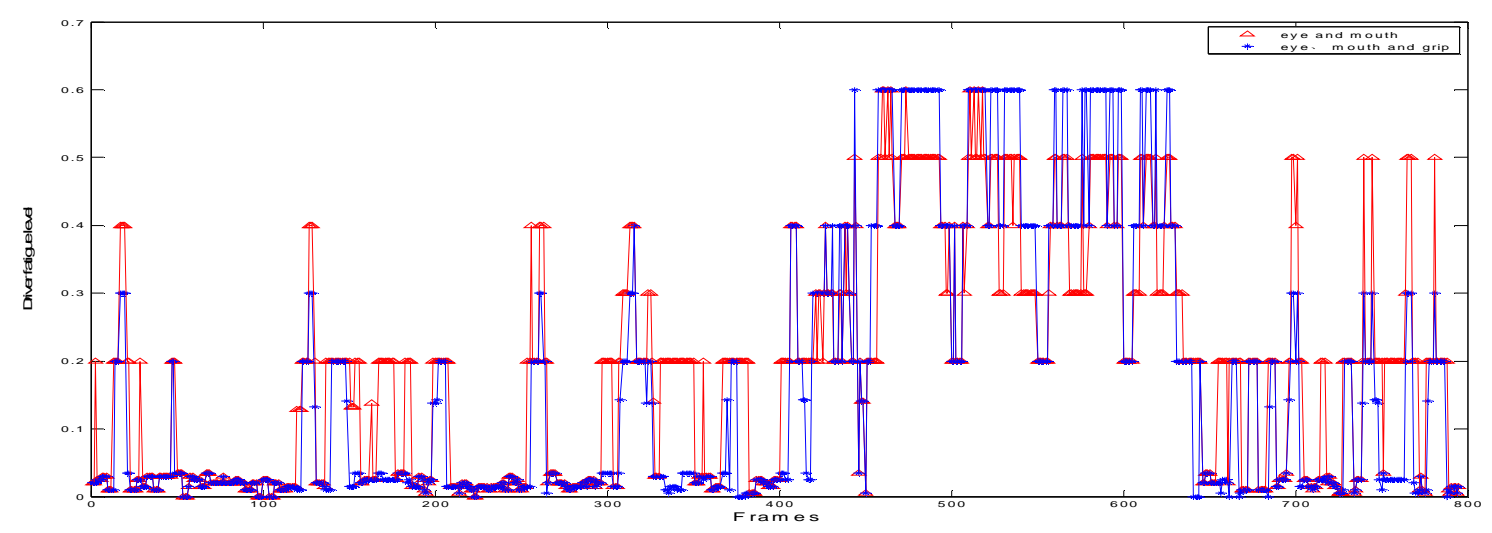

Fig. 9 Driver fatigue leve 


\section{Conclusions}

In this paper, we have proposed a drivers' fatigue detection system based on video image and steering grip force. Visual parameters are estimated such as degrees of eye closure and degrees of mouth opening by using adaptive boosting Adaboost and ASM. Steering grip force is obtained by using TekScan Grip pressure measurement system. The experiment was conducted in a simulated environment. The results show that the system can effectively detect the driver's fatigue, at the same time does well with drivers wearing glasses. In the future, on one hand, combined with physiological parameters such as EGG, EOG and Heart Rate to analyzing the deep reason for driver fatigue will be our task. On the other hand, we need collect more samples to train ASM model because of the algorithm based on statistics, and then experiments will be conducted in real driving environment to check the feasibility of the proposed method.

\section{Acknowledgements}

This work was supported in part by the Key Project of the National Nature Science Foundation of China (No. 61134009), the National Nature Science Foundation of China (Nos. 61473077, 61473078, 61503075), Cooperative research funds of the National Natural Science Funds Overseas and Hong Kong and Macao scholars (No. 61428302), Program for Changjiang Scholars from the Ministry of Education, Specialized Research Fund for Shanghai Leading Talents, Project of the Shanghai Committee of Science and Technology (No. 13JC1407500), Innovation Program of Shanghai Municipal Education Commission (No. 14ZZ067), Shanghai Pujiang Program (15PJ1400100), and the Fundamental Research Funds for the Central Universities (Nos . 15D110423, 2232015D3-32).

\section{References}

[1] J. Sousanis, in: World Vehicle Population Tops 1 Billion Units [J]. Ward's Auto World Exclusive Insight, (2011).

[2] J.A. Horne, L.A. Reyner, in: Sleep related vehicle accidents.[J]. Bmj, 310(6979):1411-1411(1995).

[3] D. Royal, in: "VOLUME I: FINDINGS -- NATIONAL SURVEY OF DISTRACTED AND DROWSY DRIVING ATTITUDES AND BEHAVIORS: 2002." Behavior (2003).

[4] R.C. Coetzer, G.P. Hancke, in: Driver fatigue detection : A survey[C]// Africon (pp.1-6),(2009).

[5] R.F. Du, R.J. Liu, T.X. Wu, and B.L. Lu, in: Online vigilance analysis combining video and electrooculography features. International Conference on Neural Information Processing (Vol.7667, pp.447-454) (2012).

[6] S.J. Jung, H.S. Shin, and W.Y. Chung, in: Driver fatigue and drowsiness monitoring system with embedded electrocardiogram sensor on steering wheel [J]. Iet Intelligent Transport Systems, 8(1):43-50 (2014).

[7] T.C. Chieh, M.M. Mustafa, and A. Hussain, in: Driver fatigue detection using steering grip force[C]// Research and Development, 2003. SCORED 2003. Proceedings. Student Conference on. (pp.45 - 48) (2003).

[8] F. Li, X.W. Wang, and B.L. Lu, in: Detection of Driving Fatigue Based on Grip Force on Steering Wheel with Wavelet Transformation and Support Vector Machine[M]// Neural Information Processing. (pp. 141-148) (2013). 
[9] T. Azim, M.A. Jaffar, and A.M. Mirza, in: Fully automated real time fatigue detection of drivers through Fuzzy Expert Systems[J]. Applied Soft Computing, 18(1):25-38 (2014).

[10] P. Viola, M.J. Jones, in: Robust Real-Time Object Detection [J]. International Journal of Computer Vision, 57(2):87 (2001).

[11]R. Lienhart, J. Maydt, in: An extended set of Haar-like features for rapid object detection. International Conference on Image Processing (Vol.1, pp.I-900-I-903 vol.1) (2002).

[12] T.F. Cootes, C.J. Taylor, in: Statistical Models of Appearance for computer vision [J]. University of Manchester, (2004).

[13] S. Milborrow, F. Nicolls, in: Active shape models with SIFT descriptors and MARS. International Conference on Computer Vision Theory and Applications (Vol.2, pp.380-387). IEEE (2014).

[14]L. Wei, S.C. Mukhopadhyay, R. Jidin, and C.P. Chen, in: Multi-source information fusion for drowsy driving detection based on wireless sensor networks. Seventh International Conference on Sensing Technology (pp.850-857) (2013).

[15]L.M. Bergasa, J. Nuevo, and M.A. Sotelo, in: Real-time system for monitoring driver vigilance [J]. Intelligent Transportation Systems IEEE Transactions on, 7(1):63-77 (2006). 